\title{
Toward the theory of strongly coupled Quark-Gluon Plasma (sQGP)
}

Edward Shuryak ${ }^{a}$

${ }^{a}$ Department of Physics and Astronomy, University at Stony Brook, NY 11794, USA

We review recent progress toward understanding of sQGP. The phenomenological part includes discussion of elliptic and conical flows at RHIC. Then we proceed to first quantum mechanical studies of manybody states at $T>T c$, the "polymeric chains" $\bar{q} \cdot g . g \ldots q$ and baryons. A new model for sQGP is a classical dynamical system, in which color vector is changed via the Wong equation. First Molecular Dynamics (MD) results for its diffusion and viscosity are reported. Finally we speculate how strong correlations in matter may help solve puzzles related to jet quenching, both the magnitude and angular distribution.

\section{Introduction}

The idea that QGP at RHIC, $T=(1-2) T_{c}$, seems to be in a strongly coupled regime (sQGP) was introduced in 2003 [1, 2, 3] and was discussed a lot at the "discovery" BNL workshop [4] and QM04. Let me start with the simplest physics point possible. Although at $T>T_{c}$ quarks are deconfined, the energy needed for separation of quarks close to $T_{c}$ is huge (see Fig 1h, up to $U \sim 4 \mathrm{GeV}$. One implication is simple: the charges simply cannot get separated until very high $T$. The second: as the ratio $U / T \sim 10$ goes into the Boltzmann exponents, any perturbative approach is completely hopeless.

Instead of going to many other arguments, let me simply list main reasons which demand such a radical change, from traditional weak coupling to strong coupling methods: 1.Collective phenomena observed at RHIC lead the hydro practitioners to view QGP as a "near perfect liquid" [ [1, 6];

2.Feshbach-type resonances due to marginal states may lead to large cross sections [2].

3.Classical e/m plasma can be a good liquid too, if sufficiently strongly coupled.

4. A close relative of $\mathrm{QCD}$, the $\mathcal{N}=4$ supersymmetric Yang-Mills gauge theory can be studied in a strongly coupled $g^{2} N_{c} \rightarrow \infty$ regime at finite $T$ via the AdS/CFT correspondence: the results are very close to what we observe at RHIC.

Since I cannot cover all these topics in depth, let me just provide a more detailed list of these arguments, with at least some statements and references:

(1a) The departure of elliptic flow data from the hydro prediction happens only at rather high $p_{t} \sim 1.5-2 \mathrm{GeV}$, from which the estimated viscosity-to-entropy ratio $\eta / s=.1-.2$ [6] is more than order of magnitude lower than in pQCD.

(1b) Another transport coefficient, the charm diffusion constant $D_{c}$ deduced from single electron $R_{A A}$ and $v_{2}$ (much discussed at this meeting), is also an order of magnitude lower than $\mathrm{PQCD}$ estimates [ [7]. 

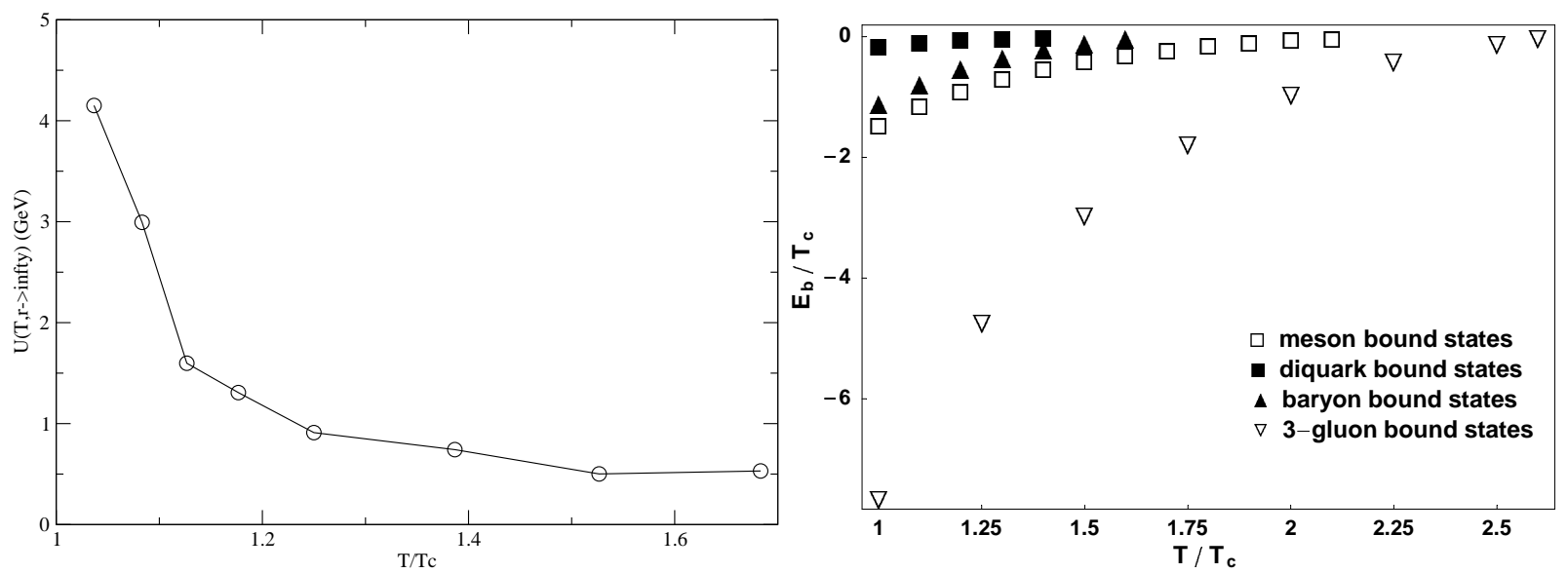

Figure 1. (a) The separation energy $U(T, r \rightarrow \infty)-U(T, r=.2 f m)$ in $G e V$ vs $T / T_{c}$, calculated from the free energy [ [5] by removing the entropy term.(b) Dependence of various states' binding energy (in units of $T_{c}$ ) on the temperature.

(1c) New hydrodynamical phenomenon suggested recently [ 8], the conical flow, maybe explains why secondaries from a quenched jet fly preferentially to large angle $\approx 70$ degrees consistent with the Mach angle for (time-averaged) speed of sound.

(2a) Combining lattice data on quasiparticle masses and interparticle potentials, one indeed finds a lot of bound states [9] and resonances [10] at $T>T c$

(2b) The same approach explains why $\eta_{c}, J / \psi$ remains bound till near $3 T_{c}$, as was directly observed on the lattice [11];

(2c) this approach was experimentally demonstrated to work for ultra-cold trapped fermionic atoms $\mathrm{Li}^{6}$, turning it to near prefect liquid as well when the Feshbach resonance leads to the scattering length $a \rightarrow \infty$. Experiments on oscillations found a sharp minimum in damping near the resonance, reducing it by about 2 orders of magnitude. According to our study [12] two lowest modes are well described by hydro with "quantum viscosity" $\eta \approx 0.3 \hbar n$, in a way nearly as low as that of sQGP.

(2d) Heavy-light resonances in sQGP can explain the value of the charm diffusion constant [13].

(3a) The interaction parameter $\Gamma=<$ potential energy $>/ T$ in sQGP is obviously not small, $\mathrm{O}(10)$. At such $\Gamma$ the classical strongly coupled e/m plasma is a good liquid: we find the same in our classical version of sQGP as well;

(4a) The EoS for finite $T \mathcal{N}=4$ SUSY YM is similar to what is seen on the lattice in the RHIC domain, namely $p / p_{\text {ideal }}=\left[(3 / 4)+O\left(\left(g^{2} N_{c}\right)^{-3 / 2}\right)\right][$ [14;

(4b)At infinite coupling there is a finite limit of viscosity $\eta / s=>1 / 4 \pi$ [ 15], again close to the RHIC value. The "R-charge" diffusion constant is low as well.

(4c) One may even think about AdS/CFT "gravity dual" to the whole RHIC collisions, with black hole production [16] which then fly away from the test brane [17]. 


\section{Systematics of collective flows}

\subsection{Elliptic flows}

Collective flows observed at SPS and RHIC are quite accurately reproduced by ideal hydrodynamics for the QGP/mixed phase, complemented by a hadronic cascade for the hadronic phase [19]. The radial flow predictions are in quantitative agreement with the data for all secondaries, from $\pi$ to $\Omega^{-}$. Elliptic flow is described by $v_{2}=<\cos (2 \phi)>$ depending on 6 variables $v_{2}\left(s, p t, M_{i}, y, b, A\right)$, the energy, transverse momentum, particle mass, rapidity, centrality and system size. Hydro works for not-too-large $p_{t}<2 \mathrm{GeV}$ (above it a different and still poorly understood regime starts.)

(i) The energy dependence was the issue I discussed already at QM99[ 18], $v_{2}(s)$ was predicted to be smoothly rising from SPS to RHIC by about factor 2, as stiffer EoS of the QGP replaces that of hadronic gas and mixed phase, see more in [19]. It was soon confirmed by the first RHIC data ${ }^{1}$.

(ii) the (pseudo)rapidity dependence $v_{2}(\eta)$ has a triangular shape (PHOBOS). Although the first 3-d hydro by Hirano et al were not able to reproduce it, now with a hydro+cascade approach [20] it works well. The physics is basically the same as for $v_{2}(s)$ : in fact $v_{2}$ displays good "limiting fragmentation", it depends mostly on local density $v_{2}(d N / d y(s, y))$ rather than on $s$ and $y$ by itself.

(iii) The $v_{2}\left(p_{t}, M_{i}\right)$ dependence for different species, known as the "fine structure" of the elliptic flow, was discussed here by R.Lacey [21] who pointed out a scaling relation $v_{2} \sim M_{i} y_{t}^{2}$ where $y_{t}$ is the transverse rapidity. Suggested by hydro, it is perfectly satisfied by the data for all secondaries.

(iv) Centrality and size dependence: ideal hydro is scale invariant and $v_{2}$ is basically given by the spatial deformation, thus $v_{2}(b, A) / \epsilon_{2}(b, A) \approx \operatorname{const}(b, A)$. This relation is in excellent agreement with the data.

Remaining hydro-skeptics include Bhalerao et al [22], who ascribe the rise in $v_{2}(s)$ (or that in $v_{2}(\eta)$ toward midrapidity) to an "incomplete equilibration". It was pointed out earlier by H.Heselberg et al [23] that hydro scaling (iv) should be violated for sufficiently peripheral collisions, by a "dilute regime". Unfortunately, due to experimental difficulties that regime was never really clearly observed. Bhalerao et al pointed out possible dependence on the system size, predicting violation of hydro scaling $v_{2}(C u C u) / v_{2}(A u A u) \sim 1 / 3$. Recent $\mathrm{CuCu}$ data do not agree with them while the hydro scaling is still satisfied.

An overall blue sky still has two clouds: (i) $v_{4} / v_{2}^{2}$ seem to be a constant, but not .5 as ideal hydro predicts, but about 1.2. It may be related to the issue of $v_{2}$ fluctuations discussed by Mrowczynski and myself [24].). (ii) the HBT radii remain a puzzle. Paradoxically. we can better describe early stages (when the elliptic flow is formed) than very late dilute ones. It may well be that our freezeout conditions via naive cascades are no good: effective potentials, resonances etc may be different than we thought ${ }^{2}$.

\footnotetext{
${ }^{1}$ Hydro with fixed freezeout, at $T_{f}$, lead to a non-monotonous curve which is not the case.

${ }^{2}$ One recent attempt, by Cramer and G.Miller, has generated discussion here. I complained that they do not account for the pions absorbed by their optical potential on the way out. B.Muller, in a summary, defended them because "the source term in the HBT formalism describes the vertex position of the last inelastic interaction". As the author of that formalism (in 1973) I cannot agree more: thus Cramer and Miller better follow their pions to that last interaction.
} 


\subsection{Conical flow}

Let me start reminding a general kinematics of the most elementary process $1=>2$, possible for a jet in matter. No change in the mass implies $0=(p-k)^{2}-p^{2}=-2(p k)+k^{2}$. Provided the last small term (recoil) can be dropped, the angle between $\vec{k}$ and $\vec{p}$ is then $\cos (\theta)=\left(k_{0} p_{0} / k p\right)$, For very fast particle $p_{0} / p=1$ and thus the condition for the process is simply $k_{0}<k$.

Now, the RHIC data on 2-particle correlation with a triggered jet show that most radiation goes into a peak at angles $60-70$ degrees, so that $\cos (\theta) \approx 1 / 3$ and thus that must be the $k_{0} / k$ ratio for what is emitted. This fits very well to sound waves, for which this ratio is the speed of sound $c_{s}$, and its average value over the duration of the collision is indeed about ${ }^{3} 1 / 3$. One simple way to test that was suggested by Antinori and myself [25]: if the jet is a b quark (which can be tagged experimentally) $p_{0} / p=1 / v>1$ which causes the cone to shrink, till it goes to zero at the critical velocity $v=c_{s}$. Gluon radiation behaves in the opposite way with decreasing $v$, and never shrinks to zero.

A number of people suggested a possibility of gluon

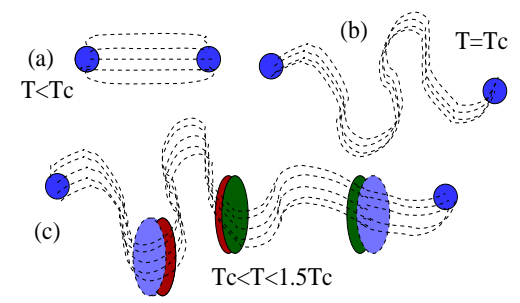
Cherenkov radiation, see the talk by Ruppert for details. There is very little support in data or theory for that: but even the optimists find the angles for Cherenkov emission of gluons with $p \sim 1 \mathrm{GeV}$ to be at least order of magnitude smaller than the observed peak at $\sim 70$ degrees.

Whether the mechanism of $d E / d x$ is a gluon radi-

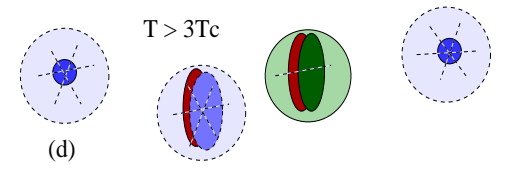
ation or scattering or ionization, a small mean free path implies rapid local deposition of energy, with subsequent hydrodynamical process. This issue is discussed in detail in the talk of my collaborator Casalderrey-Solana [ 8]: The observed shape of the

Figure 2: Schematic picture of peak, its magnitude and $p_{t}$ dependence is in rough agreement with the data. The issue of flow subtraction seem to be resolved (see B.Cole's talk), but 3particle correlations are still too controversial to comment. Let me only remind that our hydro solutions are obtained with arbitrary viscosity, and that the observed effects already constrain the viscosity more than all data on $v_{2}$ since the gradients are larger for conical than for elliptic flow.

\section{First quantum manybody states above $T_{c}$}

The existence of bound states of quark and gluon quasiparticles at $T>T_{c}$, suggested in [2] was much discussed at this meeting. Let me first report on recent studies [ 26], done in collaboration with grad.student J.F.Liao.

One result is about polymeric chains of the type $\bar{q} g g . . g q$ which appear naturally in a string picture ${ }^{4}$. For this problem the most useful coordinates in this case are not the usual Jacobi but "chain coordinates", using which we have proven that such polymers have the

\footnotetext{
${ }^{3}$ Note, we don't mean here speed of sound squared!

${ }^{4}$ Recall that a gluon has two color indices and can thus be connected to two strings.
} 
same binding energy per bond $d^{5}$ as for $\bar{q} q$ mesons. We also studied (variationally) two 3-body problems: the closed (3-)chains of gluons $(g g g)$ and $(q q q)$ baryons. Their binding energy (and range) is shown in Fig 1 one can see that the former one is quite robust while baryons have quite marginal binding (because of smaller relative charge of quarks). Before we go forward with a general discussion, let us try to summarize the proposed scenario as a single picture, see Fig 1(b). From relatively short string-like configuration of color fields at low $T$, fig (a), one moves to longer strings (b) at the critical point [ 28]. New is picture (c) which depicts "polymeric chains" considered in this work, significant at $T=(1-1.5) T_{c}$. Eventually, at high $T$, one goes into $(\mathrm{d})$ with independent quark and gluon quasiparticles, neutralized by isotropic Debye clouds.
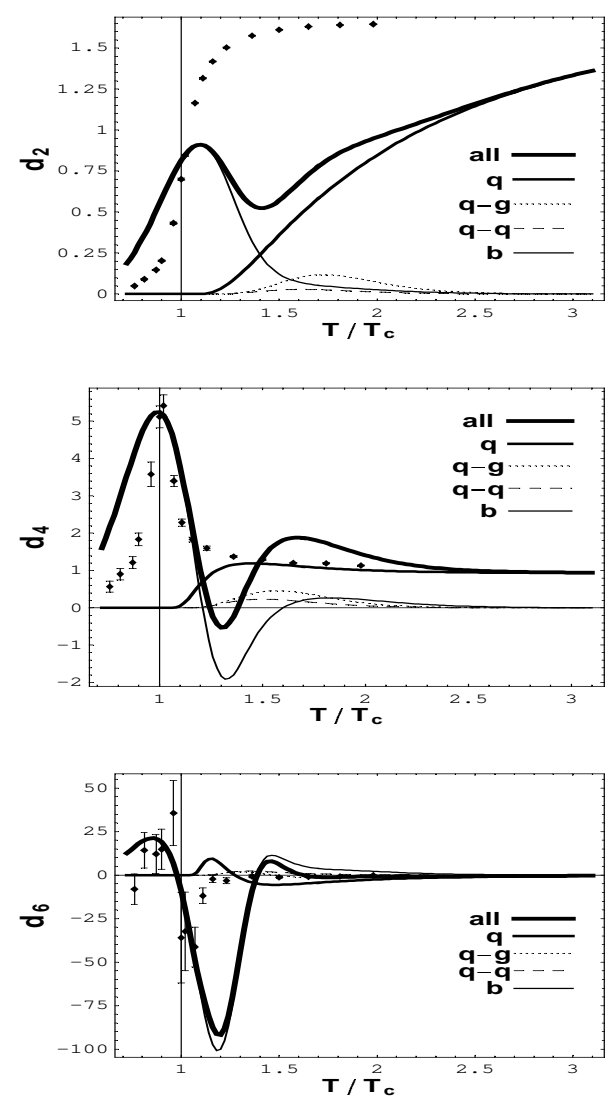

Figure 3: The contribution of different states to (a) $d_{2}$, (b) $d_{4}$ and (c) $d_{6}$ in a "bound state" model, the points are data $[32]$

Let me now briefly touch on criticism of the "bound state QGP" raised at this meeting. First, let us be clear what is being criticized. The bound states are not ad hoc ideas but the direct mathematical consequence of: (i) quasiparticle masses from the lattice [29]; (ii) lattice-based effective interaction between colored charges [5]; and (iii) quantum mechanics. None of the critics ever suggested that either (i) or (ii) or (iii) may be wrong. Where the direct test has been made, for the charmonium states [11], one finds a good agreement. And it does not take much to realize that quasiparticles with $M \sim 1 \mathrm{GeV}$ should behave like the charmed quarks.

V.Koch et al [30] and F.Karsch[ 31] argued that they do not see a significant contributions of diquarks (and baryons) in the lattice data on $d_{n}(T)=$ $\partial^{n}\left(p / T^{4}\right) /\left.\partial(\mu / T)^{n}\right|_{\mu=0}$ with $\mathrm{n}=2,4,6$ at $T>1.2 T c$. It is hardly surprising, as there are not so many states of those and the expected masses are rather large, $2 M_{q}$ and $3 M_{q}$, respectively ${ }^{6}$. (In [ 9] we have not even included diquarks and baryons in pressure, to which they clearly contribute too little.) Karsch [ 31] further argued that the ratio $\left.d_{4} / d_{2} \approx<B^{2}\right\rangle$ gives directly the mean constituent baryon number $B$, and since this ratio gets close to 1 above $T_{c}$, all bound states with $B>1$ are excluded. But the same reasoning gives $d_{6} / d_{4} \approx<B^{2}>$ as well: this ratio is however not close to 1 but $\sim-10$. Does it exclude any quark gas model as well?

In fact the argument is simply too naive and ignores a lot of things. What is worse, Karsch et al have not explained the most prominent features of his data, the peak in $d_{4}(T)$ and the "wiggle' in $d_{6}(T)$. see Fig 3 , or large

\footnotetext{
${ }^{5}$ Not per particle, so long enough chains have twice more binding per particle than mesons.

${ }^{6}$ In fact all their arguments can be repeated verbatim for say $T \sim 50 \mathrm{MeV}$ or so, with the same conclusion: no visible baryons in a hadronic gas. They do exist as states there, of course.
} 
flavor-nondiagonal $d_{4}(T), d_{6}(T)$.

It was pointed out in ref.[ 34, that since the quasiparticle masses may depend on $\mu$ their derivatives such as $M^{\prime \prime}=\left.\left(\partial^{2} M / \partial \mu^{2}\right)\right|_{\mu=0}$ must be included in all formulae. The same is true for masses of the bound states such as baryons. It is shown in our paper [33] that all above mentioned features of higher susceptibilities are naturally explained by the expected change in the baryon $(N, \Delta)$ mass $M_{B}(T, \mu)$. At the phase boundary it is expected to grow from the "vacuum mass" $\sim 1 \mathrm{GeV}$ to much larger value in sQGP, $\approx 3 M_{q}$. As a result $M_{B}^{\prime \prime}$ is large and changes sign at the inflection point near $T_{c}$ : that is why there is a peak in $d_{4}(T)$ and the "wiggle" in $d_{6}(T)$, see the baryon curve $b$ in Fig 3 , (The effect of binding of $q q$ and $q g$ states is not included in this plot: in the latter case it can increase its contribution by factor $2-3$ and make a better agreement with data, especially for $d_{2}$.)

\section{Strongly coupled colored classical plasma, studied via Molecular Dynamics}

Nice introduction to strongly coupled Abelian e/m plasmas has been provided here by M.Thoma, so let me jump directly to the point. The interaction parameter is defined as

$\Gamma=C_{c} \alpha_{s} n^{1 / 3} N_{c o r r} / T$

where the color Casimir is $C_{c}=4 / 3$ and 3 for $q$ and $g$. The quasiparticle density ${ }^{7}$ is proportional to large number of degrees of freedom in QGP $n^{1 / 3} \sim N_{d o f}^{1 / 3} T$ where $N_{\text {dof }}=$ $16+2 * 2 * 3 * N_{f} \sim 50$. The number of "strongly correlated partners" $N_{\text {corr }}$ is just 1 for binary states, 2 for polymeric chains we discussed above, but goes up to 12 for a fcc crystal (see below). Combining all, even for $\alpha_{s} \sim 1$ one finds $\Gamma>1$, possibly up to $O(10)$. Further, the structure is exponentially sensitive to $\Gamma$ as it goes to Boltzmann factor. And, as we show below, $\Gamma \sim 10$ is precisely where the plasma liquid is most perfect.

New model B.Gelman, I.Zahed and myself are proposing [35] is based on the following main assumptions: (i) The particles are heavy enough to move non-relativistically, with masses $^{8} M>>T$; (ii) Their interaction is dominated by colored electric (Coulomb) interactions, with all magnetic effects (spin forces, etc) ignored; (iii) Their color representations are large, so that color operators $t^{a}$ can be represented by classical vectors.

Dynamics of color vectors as well as particle coordinates are described by classical equations of motion $(\mathrm{EoM})^{9}$ known as Wong equation ${ }^{10}$.

$m \frac{d C^{a}}{d \tau}=g f^{a b c} p_{\mu} A_{\mu}^{b} C^{c}$

where $A_{\mu}$ is a local field induced by other particles. The total color and total energy are thus conserved (which is monitored during actual MD calculations). The interaction

\footnotetext{
${ }^{7}$ Quasiparticles inside various bound states should also be included.

${ }^{8}$ Recall that close to $T_{c}$ the $M / T$ ratio for quasiparticles reaches $4-5$.

${ }^{9}$ Another model, amenable to MD simulations, has been proposed at this meeting by the Budapest group [ 36]: but the color part is not classical and is subject to complicated stochastic transitions. Not only color is not conserved in their model, but (as far as i can tell), the energy cannot be conserved either.

${ }^{10}$ It can also be rewritten as a set of canonically conjugated equations, for one x-p pair for $\mathrm{SU}(2)$ and 3 pairs for $\mathrm{SU}(3)$, see [27] for refs. B.Muller in summary expressed doubts whether this model is gauge invariant: no reason to worry: it obviously is, this is how Wong derived it.
} 
potential is proportional to the dot product of the unit color vectors times the potential. The latter eventually should be deduced from lattice simulations, we so far use just a Coulomb. In order to stabilize the system, we have also added a short-range repulsion which is suppose to mimic the quantum-mechanical localization energy $V_{\text {short }}=\hbar^{2} / M r^{2}$, like it is sometimes done to describe systems like solid $\mathrm{He}$.

The main variable parameter of the model is obviously the temperature $T$. Eventually, in applications to sQGP all 4 parameters mentioned above are in fact some functions of $T$, so such applications of the model in the narrow sense would only be restricted to some 1-d line in a 4-d parameter space. But in order to understand them better it would be necessary first to describe the properties of the model in a wider sense, in all its parameter space.

The closest physical e/m analog of our model is the classical two-component ionic plasma, e.g. a molten ionic salts ${ }^{11}$ such as $\mathrm{NaCl}$. For large $\Gamma \rightarrow \infty$ this system freezes into the fcc cubic lattice with alternating positive and negative charges. For smaller $1<\Gamma<\Gamma_{c} \sim 80$ one deals with a strongly coupled ionic liquid. In the nonAbelian case one also expects that the system gets frozen at very large $\Gamma$, with the same cubic crystal plus quasi-Abelian ferromagnetic (alternating) order of the color vectors.

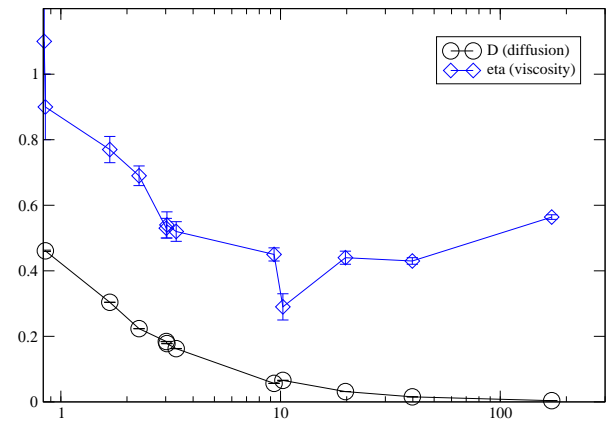

Figure 4: Our first MD data for the $\mathrm{SU}(2)$ classical plasma: the particle diffusion coefficient $D$ and viscosity $\eta$ versus $\tilde{\Gamma}=<\mid$ Pot.energy $\mid>/<$ Kin.energy $>$.
Molecular dynamics (MD) simulations can follow evolution of classical systems, weak or strong coupling. Our studies of that are at exploratory stage as of now, done so far with $4^{3}$ and $6^{3}$ particles with the SU(2) color vector (a vector on a 3 -sphere). In the QM05 talk I have shown some movies of melting a crystal by "heating" a system with a small random force: a bit of friction can cool it back. Even when pictures look homogeneous and the color vector looks isotropic, there are local correlators of neighboring particles at large $\Gamma$.

Our interest is not so far in detailed studies of its microscopic structure per se, but in collective excitations - the phonons and plasmons, as well as their widths reflecting transport properties such as the (self) diffusion coefficient $D$ and viscosity $\eta$ by Kubo formulae $^{12}$. With increased potential energy (or decreased $T$ ) the particle mobility decreases, together with $D$. But viscosity has a more interesting trend, with a minimum and subsequent rise toward a "glassy" liquid and solid regimes. This happens because the momentum transfer can be achieved not only by particle propagation but also by phonons/plasmons, which have large mean free path in perfect solids. Thus an optimum exists, with "the most perfect liquid": as Fig 4 shows, it is at $\Gamma \sim 10$ as well.

\footnotetext{
${ }^{11}$ It is a good approximation to think an electron to be completely transfered from $\mathrm{Na}$ to $\mathrm{Cl}$ and $\mathrm{T}$ still low enough not to excite other electrons.

${ }^{12}$ In fact MD is well suited for such studies, while in lattice QCD the Euclidean time makes it next to impossible to use Kubo-like formulae and access such transport properties.
} 


\section{New views on jet quenching and charm trapping}

The relevance of substructure of sQGP should be even more important for transport properties. It was first suggested by Zahed and myself in [2] that existence of marginal states must increase rescattering and thus dramatically reduce viscosity (mean free paths), leading to a collisional (hydrodynamical) regime of expansion. Since different states get marginal at different $T$, one may hope this mechanism to work at all $T$ up to about $2 T_{c}$, the highest temperature at RHIC.

We will now argue that the situation can be different for jet quenching. Its radiative theory tells that all properties of matter come one combination, $\hat{q}=5-15 \mathrm{GeV}^{2} / \mathrm{fm}$, the values currently under discussion. In most papers this value is expressed as the gluon density, with $d N / d y=1000-3000$, and some people argued this may be too large and contradict to entropy produced, see e.g. [40]. However the relation between $\hat{q}$ and $d N / d y$ assumed is simply invalid in the sQGP regime. (Think of a $\mathrm{NaCl}$-like local structure, with alternating charges: the electric field between the ions is a coherent sum of fields from positive and negative charges, which increases local fields and $\hat{q}$ compared to randomly placed charges.)

Another way to see why jet quenching (and charm equilibration) should be enhanced near $T_{c}$ is to think about collective momentum/energy sharing in multibody correlated clusters we studied above. In particular, an admixture of polymeric chains is known in material science to be very effective mechanism of the momentum distribution over larger volume of matter. A famous example is Kevlar fibers added to epoxy (or other plastic), now applied in wide range of applications, from tires, boats etc. to such exotic ones as "bullet-proof vests" and even "anti-mine boots".

If this is so, there are consequences which can immediately be checked in experiment. If indeed only a "well correlated" (polymerized?) sQGP provides large $\mathrm{dE} / \mathrm{dx}$, it should only happen at $T$ rather close to $T_{c}$. Since at RHIC the initial temperature is about $2 T_{c}$, jets may propagate with smaller losses, till the matter cools down and correlates properly. Such a delay would affect the angular distribution of the jet quenching phenomenon for non-central collisions.

In fact the original idea of "jet tomography" via jet quenching is in serious trouble for quite a while, because the most natural assumption - the energy loss proportional to the matter density - is in strong contradiction with the observed strong angular dependence of jet quenching [37], predicting too weak azimuthal asymmetry for non-central collisions. It was however recently pointed out by Pantuev [38] that a better description of data can be achieved if the jet quenching at the highest RHIC energy is switched on after some "latent time" of about $2.2 \mathrm{fm}$. This time quite reasonably matches a cooling time from $T \approx 2 T_{c}$ to $T \approx 1.5 T_{c}$ at RHIC.

\section{Status of the theory}

Studies of sQGP are developing very rapidly, with many new ideas and connections to other fields emerging daily. Instead of a summary, let me comment instead on the rather complex status of QGP theory in general.

Before RHIC there were many approaches which looked reasonable, but then RHIC data put it under severe tests. Most of them have not survived, failing to explain collective 
flows, especially the elliptic $v_{2}{ }^{13}$. It took the courage of Gyulassy and Molnar [39] to tell us that a parton cascade can only get close to the data if the cross sections are huge, $\sigma_{g g} \sim 30 \mathrm{mb}$. But if so, the number of particles interacting at any moment is large and the very concept of a cascade - free motion interrupted by scattering events - looses its meaning ${ }^{14}$. Lattice studies have found very large potentials between colored charges near and above $T_{c}$. Now a challenge to all of us is to learn how such a strongly coupled system of quasiparticles can be organized. The paradigm shift, from wQGP to sQGP, had definitely occurred. Of course, all these events created a lot of stress in theory circles.

Is the lesson learned? Well, a lot of people are in denial. Example: in the theory summary B.Muller, after briefly acknowledging a discovery of "near perfect liquid", proceeds to "new ideas" such as a parton cascade with plasma instabilities. This subject, reviewed by Mrowczynski, is indeed interesting, and recently numerical studies have shown how such instability may develop in a quasi-Abelian regime. But now we are approaching its moment of truth: its time to do simulation without boxes, with appropriate initial distribution of partons, testing whether the instabilities can indeed lead to a "little bang" and $v_{2}$. I may be wrong, but there are many reasons to think that a collisionless weakly coupled plasma will fail this test. How large gluon masses and potentials seen on the lattice can be put into negatives by feeble magnetic effects? How can those explain heavy charm quark stopping/flow, much discussed at this meeting?

The situation is well illustrated by a cartoon shown in the summary by B.Muller ${ }^{15}$, displaying a chicken afraid to cross the street. Yes, crossing from familiar wQGP to the sQGP is scary. My advice: don't be a chicken, learn to fly, be an eagle. High above there is no fear, and one can see what people do in other fields. And, last but not least, there is basically no alternative: one can sell non-working theories only for so long.

\section{REFERENCES}

rog. Part. Nucl. Phys. 53, 273 (2004) [ hep-ph/0312227.

ak,Prog.Part.Nucl.Phys.53:273-303,2004, hep-ph/0312227 Nucl. Phys. A 750, 64 (2005).

olana's talk

atsuda, Nucl. Phys. A715 (2003) 863c; hep-lat/0308034;

fect Liquid," nucl-th/0410067.

and A.A. Tseytlin, Nucl. Phys. B534 (1998) 202

\footnotetext{
${ }^{13}$ String models (RQMD etc) get it factor 3-4 too small, and predicted its decrease with $s$. "Naive" parton models like HIJING even predicted a bit negative values, and the classical color glass fields got the $p_{t}$ dependence wrong. Parton cascade from Duke never even reported their $v_{2}$, as far as I know.

${ }^{14}$ Molecular Dynamics takes its place in the classical context.

${ }^{15}$ Although he used it in a somewhat different context, I think subconsciously he had my interpretation in mind.
} 
52 .

$106(2002) 513$.

, diquarks and baryons at $T>T c$ ? hep-ph/0510110

iangyong Jia, Phys.Rev.C71:034909,2005 e-Print Archive: nucl-th/0310044 893] 